\title{
3D Surface Reconstruction of Spinal Ultrasound Images Based on 2D Contour Line
}

\author{
Wang Jiang ${ }^{1, a}$, Junhua Zhang ${ }^{2, b}$ \\ ${ }^{1}$ Shool of Information Science and Engineering, Yunnan University, Kunming, 650091, China \\ ${ }^{2}$ Shool of Information Science and Engineering, Yunnan University, Kunming, 650091, China \\ aemail: wangjiang7152@163.com, bemail: jhzhang@ynu.edu.cn
}

\section{Keywords: Ultrasound Images; Spine; 3D Reconstruction; 2D Contour Line}

\begin{abstract}
Methods of 3D reconstruction of medical images mainly included surface and volume reconstruction at present. In the experiment of 3D ultrasound reconstruction for spine of people, the result using volume reconstruction was unsatisfactory because of the ultrasound images' characteristic. So, we brought forward a method which was based on contour line to reconstruct 3D surface of spine. In this paper, we collected spinal ultrasound images using 3D freehand ultrasound firstly; next, tracing spinal contour line of each ultrasound images manually; finally, we used these contour lines to reconstruct spinal surface. The result showed that spinal surface reconstruction based on 2D contour line was close to real spinal surface, it could give physicians a better help in diagnosis of the spine.
\end{abstract}

\section{Introduction}

It was important for medical images in medical diagnosis, 2D ultrasound images [1] were widely used in medical diagnosis, because of its no radiation, flexible and low cost. It was based on 2D ultrasound images in Traditional clinical medicine to examine body organs popularly. The doctor need estimate physical property of organs to give therapeutic method. 2D ultrasound images were no doubt positive for diagnosis, but they only appear body organs' fracture surface and cannot reflect organs' massive structure [2]. Because the dissections of body organs are 3D, doctors were based on experience to diagnose organs, and the result of diagnosis were possible not accuracy. If body organs were direct presented by $3 \mathrm{D}$, it was useful for doctors to give overall and accurate analytical result. So the research of body organs' 3D ultrasound [3] had important practical significance.

Now, the technology of 3D ultrasound was divided into two types [4,5]: direct form and indirect form. The probe in direct 3D ultrasound had small scanning scope and high cost, its use was limited. The indirection form was a freehand 3D ultrasound mainly; it became main direction of research because of flexibility and low cost. The system of freehand 3D ultrasound was convenient to be constituted, it need a position sensor and receiver of sensor was taped to the traditional ultrasound probe, this position sensor collected location information of corresponding images, image data and corresponding location information were inserted $3 \mathrm{D}$ voxel space to get $3 \mathrm{D}$ voxel data. The data were then drawn by surface rendering [6,9] or volume rendering [7,9], so organs' 3D images were displayed. Surface rendering achieves visualization of object surface, it mainly included MC and contour lines method; volume rendering got projected image based on optical model of 3D data field, and its method included ray casting, splatting algorithm and shear-warp algorithm.

In the $3 \mathrm{D}$ ultrasound research of body spine, the 3D reconstruction result by volume rendering is bad, because spinal 2D ultrasound images are not good, they cannot well display spinal slice contour. So, we presented a surface rendering method which was based on 2D contour line in this paper. Firstly, we used freehand 3D ultrasound system [4,5] to collect human spine image; secondly, drawing spinal contour line for each image manually; last, we used these contour lines [9] to reconstruct spinal surface. In this paper, we introduced the 3D freehand ultrasound and described its theory, next, we gave the method and step of images' collection, and then we introduced the 3D reconstruction based on 2D contour line and gave its theory, method and experiment. At length, we 
got experimental result.

\section{Collection of Spinal Ultrasound Images}

Equipment. Spinal ultrasound images were collected by freehand 3D ultrasound in our experiment. The main equipment consisted of ultrasound (the model is Mindray DP-50), position sensor (PATRIOT) and computer, as shown in Figure 1.
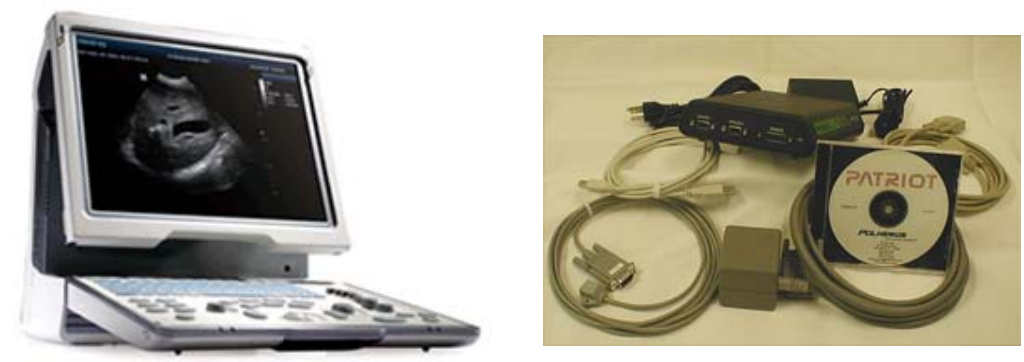

Fig.1. Mindray DP-50 ultrasound, PATRIOT

Freehand 3D Ultrasound System. Freehand 3D ultrasound system consisted of ultrasound, sensor and computer, the receiver of sensor was taped to the ultrasound probe. Ultrasound collected 2D ultrasound images data and sensor collected location information of corresponding images, we can use images data and location data to complete 3D reconstruction. The imaging process of freehand 3D ultrasound system fell into three steps [10]: scanning, reconstruction, visualization. Scanning stage obtained images data and location data; reconstruction stage was to insert the 2D images into the volume space to get $3 \mathrm{D}$ volume data $[13,14,15]$; visualization stage was to show $3 \mathrm{D}$ volume data based on surface or volume rendering. We can observe organs from arbitrary angle when organs were displayed by $3 \mathrm{D}$, the diagnostic message was more complete than $2 \mathrm{D}$ ultrasound images. Figure 2 illustrates the system's overall process.

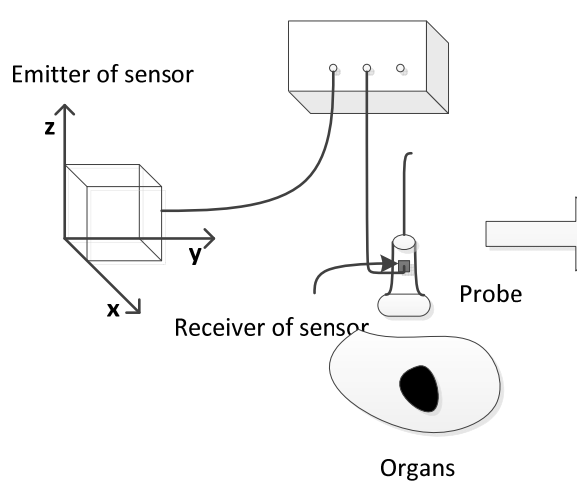

Scanning

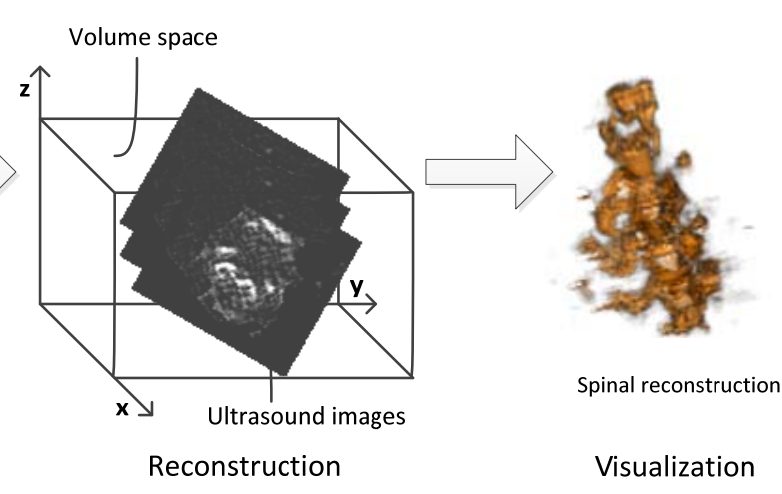

Fig.2. Freehand 3D ultrasound system

Calibration of Ultrasound Probe. The primary step is to calculate the position coordinates of each pixel in volume space. It needs turn pixel coordinates of 2D images into the volume space. So, 4 coordinate systems are introduced, as shown in Figure 3.

In the Figure 3, arrows express the direction of coordinate transformation; $\mathrm{P}$ is the coordinate system in scanning plane; $\mathrm{R}$ and $\mathrm{T}$ are the coordinate systems of sensor's receiver and transmitter; $\mathrm{C}$ is the coordinate system of volume space; The transformational relation of 2D image's pixels which from $\mathrm{P}$ coordinate system to $\mathrm{C}$ coordinate system is can be express by Eq. 1 .

$$
\varepsilon_{\mathrm{X}}-{ }^{\mathrm{s}} \mathrm{T}_{\mathrm{T}} \mathrm{T}_{\mathrm{T}_{\mathrm{R}}}{ }^{\mathrm{k}} \mathrm{T}_{\mathrm{p}}{ }^{p} \mathrm{~K}
$$

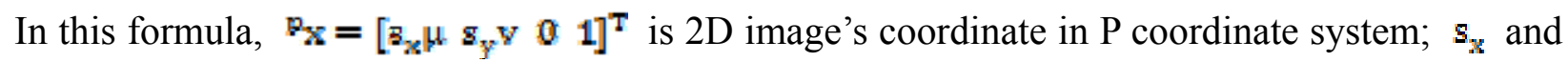


$s_{y}$ are image's resolution from portrait and landscape; $\mu$ and $v$ express pixel index; ${ }^{R} T_{p}$ is the transition matrix from $P$ coordinate system to $R$ coordinate system; ${ }^{2} T_{\mathbb{R}}$ is the transition matrix from $\mathrm{R}$ coordinate system to $\mathrm{T}$ coordinate system; ${ }^{\mathrm{C}} \mathrm{T}_{\mathrm{T}}$ is the transition matrix from $\mathrm{T}$ coordinate

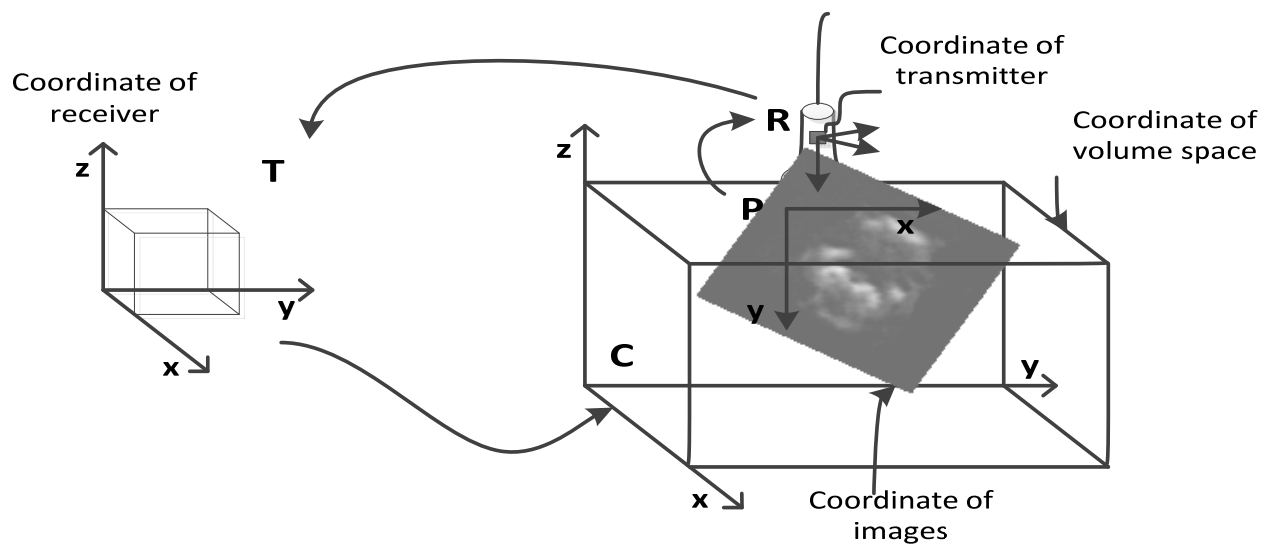

Fig.3. Transformational relation of 3D ultrasound

system to $\mathrm{C}$ coordinate system; $\mathrm{C}_{\mathrm{X}}$ is the coordinate of pixel in volume space. The three transition matrixes consist of six parameters $(x, y, z, \alpha, f, \gamma)$, the Eq. 2 is transition matrix.

$$
\mathrm{J}_{\mathrm{T}}=\left(\begin{array}{cccc}
\cos \alpha \cos \beta & \cos \alpha \sin \beta \sin \gamma-\sin \alpha \cos \gamma & \cos \alpha \sin \beta \cos \gamma+\sin \alpha \ln \gamma & \mathrm{x} \\
\sin \alpha \cos \beta & \sin \alpha \sin \beta \sin \gamma+\cos \alpha \cos \gamma & \sin \alpha \sin \beta \cos \gamma-\cos \alpha \sin \gamma & y \\
-\sin \beta & \cos \beta \sin \gamma & \cos \beta \cos \gamma & \mathbf{z} \\
0 & 0 & 0 & 1
\end{array}\right)
$$

It is difficult to ensure transformational relation between scanning plane and receiver coordinate system, so ${ }^{\mathbb{R}_{\mathrm{p}}}$ is unknown, the transition matrix need to be ensured by calibration $[11,12]$. The model which is used in calibration has point phantom and wall phantom. Take the wall phantom, wall phantom (transparent PVC plate was used in our experiment) was put in flume, we used probe to scan the phantom according to a certain manner. The wall phantom imaged a bright line, if $\mathrm{x}-\mathrm{O}-\mathrm{y}$ plane of $\mathrm{C}$ coordinate system and the bottom of flume are coincident, the point in line satisfy the Eq. 3.

$$
\left[\begin{array}{lll}
\mathrm{x} y & 0 & 1
\end{array}\right]^{\mathrm{T}}={ }^{\mathrm{Q}} \mathrm{T}_{\mathrm{T}}{ }^{\mathrm{T}} \mathrm{T}_{\mathrm{R}}{ }^{\mathrm{R}} \mathrm{T}_{\mathrm{p}}\left[\begin{array}{lll}
\mathrm{s}_{x} \mu \mathrm{s}_{\mathrm{y}} \mathrm{v} & 0 & 1
\end{array}\right]^{\mathrm{T}}
$$

${ }^{\mathrm{T}} \mathrm{T}_{\mathrm{R}}$ is ensured by the location information received from receiver; ${ }^{\mathrm{C}} \mathrm{T}_{\mathrm{T}}$ is ensured by the volume space; letting the point in line and its corresponding location information into the Eq. 3 to get a nonlinear equation. Two points are taken in a line, so, if there are $n$ images, we can get $2 n$ nonlinear equations. Then, the Levenberg-Marquardt algorithm is used to solve the equations, so, ${ }^{\mathrm{T}} \mathrm{T}_{\mathrm{R}}$ can be estimated. We can ensure the location relations between probe and receiver of sensor after calibration. The information of 2D image's pixels was being into the Eq. 1 to calculate the location of each pixel in volume space.

Method and Steps of Image's Collection. Don't change the location between probe and sensor after calibration, then, scanning body spine to get spinal images. In order to ensure the accuracy of $3 \mathrm{D}$ reconstruction, probe need keep level and scan from up to down or down to up. The steps are as follows.

(1) The spine was scanned by the ultrasound which is calculated, collecting spinal ultrasound images video by Data Acquisition Card and getting the location data by PATRIOT.

(2) Spinal ultrasound images video was converted into bmp images, it can set your own frequency (for example, a second video was turned into 20 images).

(3) Making each ultrasound images correspond each location data based on image's frequency and location data's frequency. Then, we can calculate the location of each image in volume space. 


\section{Reconstruction Based on 2D Contour Line}

Principle. Reconstruction based on 2D contour line [9] is to connect the Contour points of the target in two adjacent slice images with a certain rule. Forming a set of triangular facets, then, polyhedral approximation of the surface of the reconstructed object is formed. As shown in figure 4 and figure 5 .

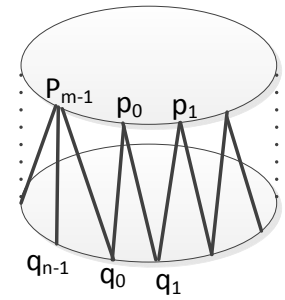

Fig.4. Reconstruction grid of contour line

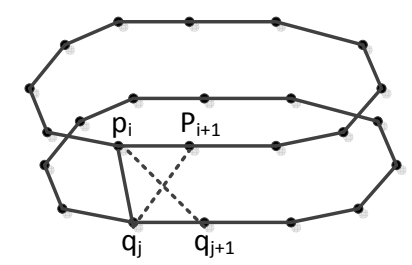

Fig.5. Sketch of the shortest diagonal method

Suppose the contour lines in the two slice images are $\mathrm{P}$ and $\mathrm{Q}$, the vertex sequence of upper contour line consist of $\mathrm{p}_{0}, \mathrm{p}_{1}, \cdots, \mathrm{p}_{\mathrm{m}-1}$, the lower consist of $\mathrm{q}_{0}, \mathrm{q}_{1}, \cdots, \mathrm{q}_{\mathrm{n}-1}$. The vertices on the contour line are arranged in a counter clockwise direction, then, the contour vertices in each slice image are connected in a straight line in turn, so, we can get polygon approximation. Each line segment $\mathrm{p}_{\mathrm{i}} \mathrm{p}_{\mathrm{i}+1}$ or $\mathrm{q}_{\mathrm{j}} \mathrm{q}_{\mathrm{j}+1}$ are called the contour line segment, and straight line segment connecting the upper and lower contour vertices called span. In order to make the 3D surface don't intersect, the triangle should meet the following three conditions.

(1) The three vertices of the triangle must locate in both P and Q.

(2) Each contour line segment must be a side of a triangle.

(3) A span can only belong to the two adjacent triangles.

If the upper and lower contour lines have $\mathrm{M}$ and $\mathrm{N}$ line segment, there are $\mathrm{M}+\mathrm{N}$ basic triangles. Triangles according with the above 3 conditions can have many different combinations, in order to ensure the smooth of the surface, some optimization criteria are used to constrain. Optimization criteria commonly used consist of maximum volume criterion, minimum surface area criterion and the shortest span criterion. In this paper, we used a shortest span criterion to realize spinal 3D surface reconstruction that is a shortest diagonal method. This method is simple and easy to implement, Algorithm procedure is as follows.

(1) The vertices of the contour lines are sorted by counter clockwise.

(2) Selecting a point $\mathrm{q}_{\mathrm{j}}$ as a starting point on the lower contour line, calculating the distance from the point on upper contour line to the $\mathrm{q}_{\mathrm{j}}$, selecting the nearest point from $\mathrm{q}_{\mathrm{j}}$, assuming this point is $\mathrm{p}_{\mathrm{i}}$, and then $\mathrm{q}_{\mathrm{j}}$ and $\mathrm{p}_{\mathrm{i}}$ are the two vertices of the reconstructed triangle.

(3) Calculating the distance between $\mathrm{q}_{j}$ and $\mathrm{p}_{i+1}$, recorded as $\mathrm{D}_{1}$, and the distance between $\mathrm{p}_{i}$ and $\mathrm{q}_{\mathrm{j}+1}$, recorded as $\mathrm{D}_{2}$.

(4) If $D_{1}<D_{2}$, then the third vertices of the triangle is $p_{i+1}$, forming a triangle $p_{i} q_{j} p_{i+1}$, updating $\mathrm{i}=\mathrm{i}+1$; otherwise, the third vertices of the triangle are $\mathrm{q}_{\mathrm{j}+1}$, forming a triangle $\mathrm{q}_{j} \mathrm{p}_{\mathrm{i}} \mathrm{q}_{\mathrm{j}+1}$, updating $j=j+1$. Then, turning step (3), around all the vertices of the contour for a week, to determine all the vertices of the triangle.

Method. In order to test the feasibility of 2D parallel contour line algorithm for 3D reconstruction of spine, the spine model and the real human spine were used to do 3D reconstruction experiment in this paper, spinal ultrasound images were collected by freehand 3D ultrasound. We scanned the spine model which is put into the bottom of water to get ultrasound images. The collection method of real body spine was the same as hospital. We described the contour line of each ultrasound image manually, and then these 2D contour images were reconstructed by 2D parallel contour line algorithm. The process is as shown in Figure 6. 


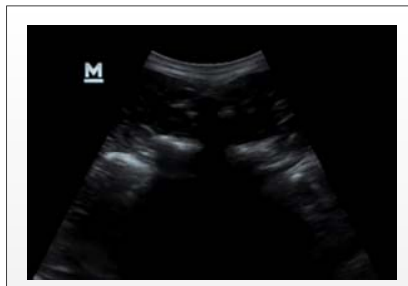

(a) Ultrasound images

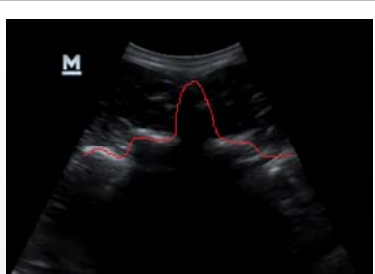

(b) Contour line

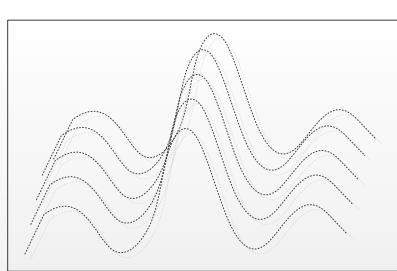

(c) Contour lines are inserted into volume space

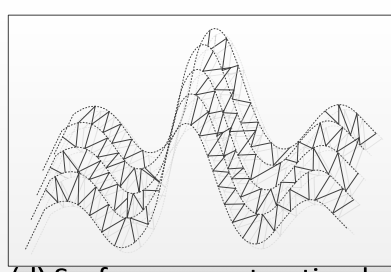

(d) Surface reconstruction by contour line

Fig.6. Process of spinal surface reconstruction

In the figure 6, (a) is spinal ultrasound images, we analyzed the characteristic information of the spinal ultrasound image, and contour line of the spine is described according to the information manually. (b) is spinal contour line, a series spinal contour lines are inserted into volume space layer by layer, as shown in Figure (c). Finally, the surface reconstruction method of contour line is used to reconstruct spine, the spinal contour, getting the spinal surface contour as shown in Figure (d).

Result. A set of spine model and a young human spine were as the experimental object in this paper, 150 spine model ultrasound images and 150 real human spine ultrasound images were collected respectively. We described the contour line of each image and reconstructed the spinal surface based on 2D contour line method. Reconstruction results are shown in Figure 7.

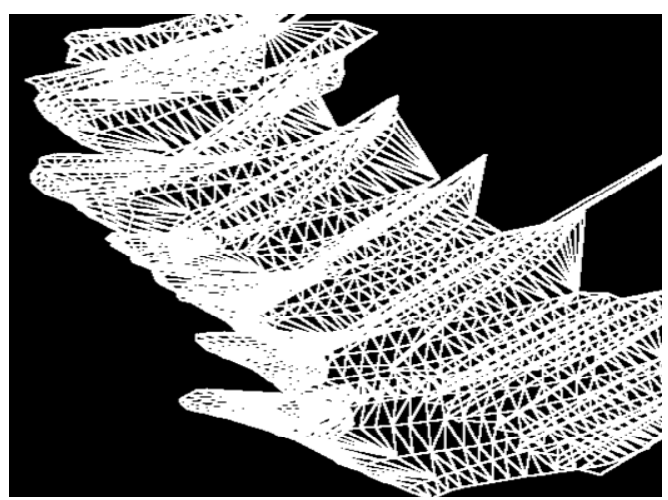

(a) Reconstruction result of spine model

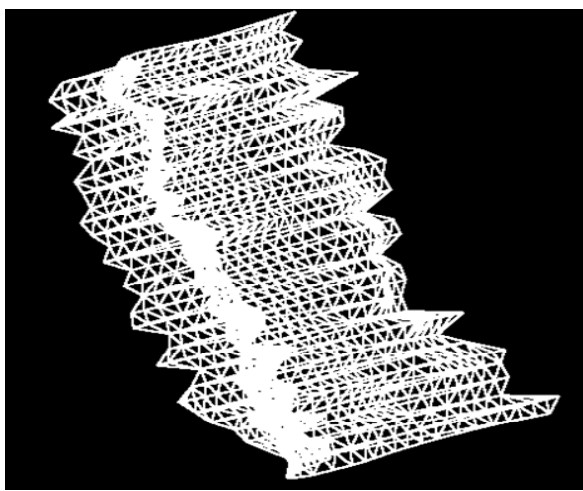

(b) Reconstruction result of real spine

Fig.7. Reconstruction result of spine

In the figure 7, (a) is reconstruction result of spine model; (b) is reconstruction result of real body spine. Observed the two reconstruction results, we found the two reconstruction results reflect their original spine surface. But the reconstruction result of spine model was better than the real body spine's, its surface features more were close to the original spine model. There are three reasons to according to this phenomenon: Firstly, the scanning methods of the two kinds of experiment objects are different. The spine model was scanned in the bottom of water, its ultrasound images were good and spine contour is more obvious; on the contrary, the scanning of the real spine was influenced by the body organs, its ultrasound images were bad and spine contour is not obvious. Secondly, when described the contour line, we depended on experience, the contour line was influenced by artificial. Finally, ultrasound equipment is also an important factor affecting the results of reconstruction. Even so, we can improve the algorithm further and the quality of contour line to get better spine surface contour. It can provide help for doctors to diagnose the spine.

\section{Conclusion}

With the development of medical imaging technology, medical imaging techniques such as ultrasound, CT, and nuclear magnetic resonance were invented. These techniques improved the level of medical diagnosis greatly. The CT images are also better than ultrasound images, but the high cost prevents its wide range of use. So, most of medical diagnostic images are ultrasound images. In order to improve the diagnostic level of ultrasonic technology, we can reconstruct the 2D 
ultrasound images and get the organs' 3D contour, because of its low cost and convenience. In this paper, we reconstructed spine contour with 2D contour line method, the result is good, but it has a gap with the real spine. We hope to improve the quality of ultrasound and optimized algorithm later, so, the quality of $3 \mathrm{D}$ reconstruction of spine will be improved.

\section{References}

[1] Vidya K Sudarshan, Muthu Rama Krishnan Mookiah. Application of wavelet techniques for cancer diagnosis using ultrasound images: A Review [J]. Computers in Biology and Medicine, 2016, 69(1):97-111.

[2] Fenster A, Downey DB, Cardinal HN. Three-Dimensional Ultrasound Imaging [J]. Physics in Medicine and Biology, 2001, 46(5): R67-R99.

[3] Peng Gu, Won-Mean Lee, Marilyn A. Automated 3D ultrasound image segmentation to aid breast cancer image interpretation [J]. Ultrasonics, 2016, 65:51-58.

[4] Qinghao Wang. Research on the key technology of navigation system of freehand 3D ultrasound [D]. Jinan: Shandong University, 2010.

[5] Xulei Jiang. Real time 3D diagnosis and navigation of freehand ultrasound [D]. Shanghai: Shanghai Jiao Tong University, 2009.

[6] D.Cebrian-Robles, J.Ortega-Casanova. Low cost 3D underwater surface reconstruction technique by image processing [J]. Ocean Engineering, 2016, 113:24-33.

[7] Xiaoqi Lv, Yu Miao, Xiaoying Ren, Jianshuai Wu. The study and implementation of liver volume measuring method based on 3-dimensional reconstruction technology [J]. Optik International Journal for Light and Electron Optics, 2015, 126(17):1534-1539.

[8] Duc V.Nguyen, Quang N.Vo, Lawrence H.Le. Validation of 3D surface reconstruction of vertebrae and spinal column using 3D ultrasound data - Apilot study [J]. Medical Engineering and Physics, 2015, 37(2): 239-244.

[9] Junhua Zhang, 3D medical image reconstruction and visualization - VC++ instances of the implementation [M]. Beijing: Science Press, 2014.

[10] Yakang Dai, Tianjie. Freehand 3D ultrasound of real-time interactivity [J]. Journal of Software, 2016: 28-37.

[11] Francois Rousseau, Pierre Hellier, Christian Barillot. Confhusius: A robust and fully automatic calibration method for 3D freehand ultrasound [J]. Medical Image Analysis, 2005, 9:25-38.

[12] R.W.Prager, A.H.Gee, L.Berman. Stradx: real-time acquisition and visualization of freehand three-dimensional ultrasound [J]. Medical Image Analysis, 1999, 3(2):129-140.

[13] Robert Rohling. 3D Freehand Ultrasound: Reconstruction and Spatial Compounding [J]. Thesis. University of Cambridge, 1998.

[14] M.Nakamoto, Y.Sato, K.Nakda. A Temperal Calibration Method for Freehand 3D Ultrasound System a Preliminary Result [C]. International Congress Series, 2003.

[15] Qing-Hua Huang, Yong-Ping Zheng. An adaptive squared-distance-weighted interpolation for volume reconstruction in 3D freehand ultrasound $[\mathrm{J}]$. Proceedings of Ultrasonics International and World Congress on Ultrasonics(WCU), 2006, 44(1):e73-e 77. 